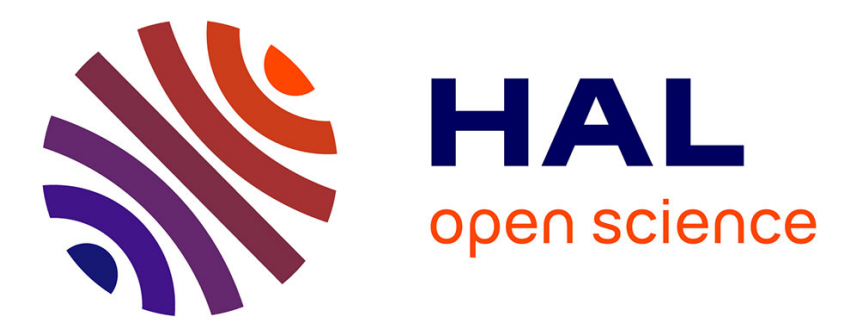

\title{
Holistic Statistical Open Data Integration Based On Integer Linear Programming
}

\author{
Alain Berro, Imen Megdiche-Bousarsar, Olivier Teste
}

\section{To cite this version:}

Alain Berro, Imen Megdiche-Bousarsar, Olivier Teste. Holistic Statistical Open Data Integration Based On Integer Linear Programming. IEEE 9th International Conference on Research Challenges in Information Science (RCIS 2015), May 2015, Athènes, Greece. pp.468-479, 10.1109/RCIS.2015.7128908 . hal-01295242

\section{HAL Id: hal-01295242 \\ https://hal.science/hal-01295242}

Submitted on 30 Mar 2016

HAL is a multi-disciplinary open access archive for the deposit and dissemination of scientific research documents, whether they are published or not. The documents may come from teaching and research institutions in France or abroad, or from public or private research centers.
L'archive ouverte pluridisciplinaire HAL, est destinée au dépôt et à la diffusion de documents scientifiques de niveau recherche, publiés ou non, émanant des établissements d'enseignement et de recherche français ou étrangers, des laboratoires publics ou privés. 


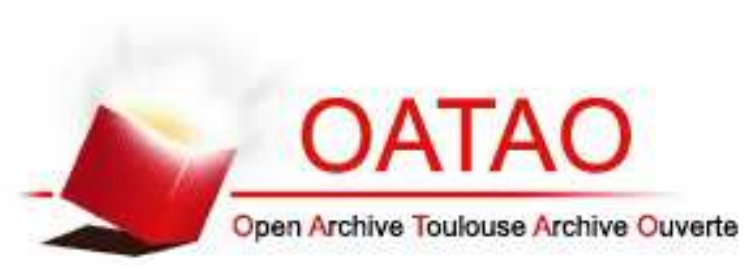

\title{
Open Archive TOULOUSE Archive Ouverte (OATAO)
}

OATAO is an open access repository that collects the work of Toulouse researchers and makes it freely available over the web where possible.

This is an author-deposited version published in : http://oatao.univ-toulouse.fr/ Eprints ID : 15292

\section{To link to this article :}

URL : $\underline{\text { http://dx.doi.org/10.1109/RCIS.2015.7128908 }}$

\author{
To cite this version : \\ Berro, Alain and Megdiche-Bousarsar, Imen and Teste, Olivier \\ Holistic Statistical Open Data Integration Based On Integer Linear \\ Programming. (2015) In: IEEE 9th International Conference on \\ Research Challenges in Information Science (RCIS 2015), 13 May \\ 2015 - 15 May 2015 (Athens, Greece)
}

Any correspondence concerning this service should be sent to the repository administrator: staff-oatao@listes-diff.inp-toulouse.fr 


\title{
Holistic Statistical Open Data Integration Based On Integer Linear Programming
}

\author{
Alain Berro, Imen Megdiche, Olivier Teste \\ IRIT UMR 5505, University of Toulouse, CNRS, INPT, \\ UPS, UT1, UT2J \\ 31062 TOULOUSE Cedex 9, France \\ \{Alain.Berro, Imen.Megdiche, Olivier.Teste\}@irit.fr
}

\begin{abstract}
Integrating several Statistical Open Data (SOD) tables is a very promising issue. Various analysis scenarios are hidden behind these statistical data, which makes it important to have a holistic view of them. However, as these data are scattered in several tables, it is a slow and costly process to use existing pairwise schema matching approaches to integrate several schemas of the tables. Hence, we need automatic tools that rapidly converge to a holistic integrated view of data and give a good matching quality. In order to accomplish this objective, we propose a new 0-1 linear program, which automatically resolves the problem of holistic OD integration. It performs global optimal solutions maximizing the profit of similarities between OD graphs. The program encompasses different constraints related to graph structures and matching setup, in particular 1:1 matching. It is solved using a standard solver (CPLEX) and experiments show that it can handle several input graphs and good matching quality compared to existing tools.
\end{abstract}

Keywords-Schema Matching, Linear Programming, Statistical Open Data

\section{INTRODUCTION}

Crossing and analysing Statistical Open Data (SOD) in data warehouses is a promising issue. However, the characteristics of SOD make them unaffordable with traditional ETL (ExtractTransform-Load) processes. Indeed, an important part ${ }^{1}$ of SOD holds into spreadsheets disposing structurally and semantically heterogeneous tables. Moreover, these sources are scattered across multiple providers and even in the same provider, which hampers their integration.

To cope with these issues, we have proposed a graphbased ETL approach composed of three steps which adapt the extract, transform and load traditional ETL steps. The first step extracts, annotates and transforms the SOD tables into a unified graph representation [1]. The second one gives a solution to the automatic holistic data integration problem through the Integer Linear Programming (ILP) technique. The third step focuses on an incremental multidimensional schema definition from the integrated graphs.

In this paper, we focus on the second phase of our ETL processes by resolving the problem of schema matching. According to [2], transformations (in ETL) can be defined from the correspondences between schema elements resolved

\footnotetext{
${ }^{1}$ http://fr.slideshare.net/cvincey/opendata-benchmark-fr-vs-uk-vs-us. The percentage of flat OD sources is $82 \%$ in France providers, 65\% in United States providers and $66 \%$ in United Kingdom providers.
}

by a matching task. Furthermore, the number of input schemas declines the schema matching problem into pairwise (2 input schemas) and holistic (several input schemas) problems [2]. Our proposition falls within the holistic schema matching approaches. It consists of an integer linear program reducible to the weighted graph matching problem and extends this latter with different constraints on graph structures and matching setup.

\section{A. An overview of the Graph-Based ETL Approach}

Our approach aims to offer users an easy and repetitive way to reuse SOD in different analysis scenarios by bringing automatic solutions in the ETL processes. Our processes are based on the idea of manipulating graphs. Different reasons motivated our choice: (i) graph data models [3] have gained attention from different research communities in previous years; (ii) graphs are flexible as they hold objects and relationships varying from the most generic and simple to the more specific and complex. Our approach [4] takes as input flat SOD spreadsheets and generates as output multidimensional schemas [5]. It involves three main steps as depicted in Fig.1:

- "Extraction and Annotation": the first step takes as input flat SOD spreadsheets and provides unified instance-schema graphs. In [1], we have proposed a solution to perform this step. The idea consists of exploiting and enriching table anatomies in order to automatically define the schema of tables. The spreadsheets contain tables which are composed of: (i) numerical data (the statistics or numerical values in tables) and (ii) structural data (the text surrounding tables in rows or columns). We have encoded the cell types into matrices, in which we apply several algorithms to extract the different blocks composing a table. These blocks are annotated according to three overlapping types. For more details refer to [1]. Two classification approaches have been applied in order to transform structural data into hierarchies. The extracted and annotated parts are transformed into a unified instance-schema graph representation. The latter is stored in a staging area.

- "Holistic Integration": the second step takes as input structural parts of several graphs and generates as output an integrated graph and the underlying matchings. The integrated graph is composed of collapsed and 


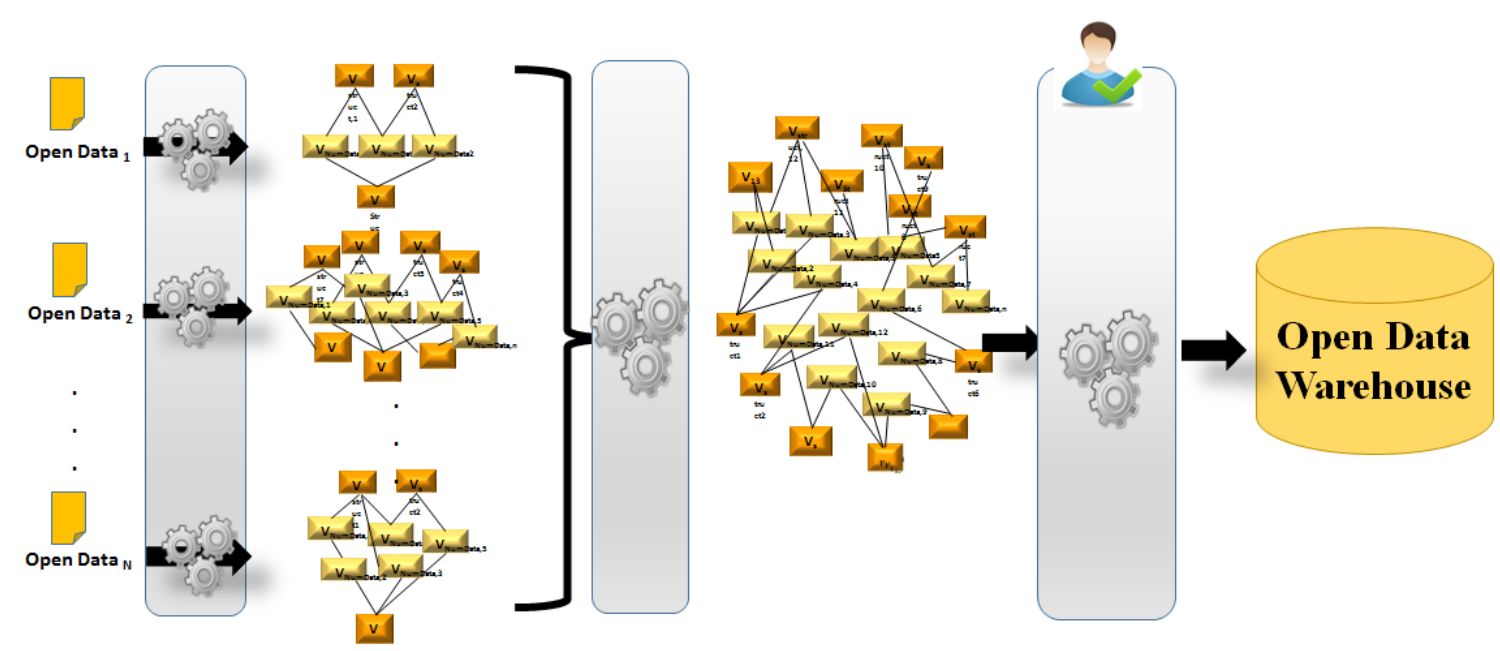

(1) Extraction and

(2) Holistic Integration Annotation

(3) Incremental Multidimensional schema definition

Fig. 1. A Graph-Based ETL Approach

simple vertices connected by simple edges. Collapsed vertices reference groups of matched vertices. Nonmatched ones remain simple vertices. We propose an integer linear program to perform an automatic and optimal holistic integration. Users can validate and/or adjust the proposed solution. It maximizes semantic and syntactic similarity between the labels of the vertices of all inputted graphs. It also resolves conflicting situations by ensuring strict hierarchies [6] and preserving logical edges' directions.

- "Incremental Multidimensional Schema Definition": the final step takes as input an integrated graph and generates a multidimensional schema. An interactive process is established between users' actions and system:

- users' actions consist of identifying multidimensional components such as dimensions, parameters, hierarchies, facts and measures. These actions are defined in increment. Users should begin by identifying the dimensions and their components (hierarchies and parameters). Then, they define the fact and its measures forming a multidimensional schema.

- the system interacts with users' actions, first, by transforming the integrated graph into a multidimensional schema. Next, it generates a script describing the multidimensional schema, from which the data warehouse is populated with information.

\section{B. Motivating Example}

An agricultural company in England aims to launch a new project on cereal production. The company is interested in analysing England cereal production activity in the previous years. The study consists of reusing SOD from one or different providers. Some data were collected from the World Bank provider. This reference shares more than 1,000 statistical indicators by topic, year and country. For the "Agriculture \& Rural Development" 2 topic, the company noticed some relevant indicators, like cereal yield or crop production. Hence, the spreadsheets ${ }^{3}$ corresponding to these indicators for the UK have been selected for the analysis study. Since the World Bank statistics are aggregated, the company collected other sources to deepen the study. They proposed to use the SOD from the UK governmental provider ${ }^{4}$. The latter provides several detailed sources for cereal production by cereal type, farm size, type, etc. Our approach perfectly meets this case study for the following reason. For several heterogeneous SOD sources, a rapid and automatic integration is advantageous to this company. They will conserve resources and time by avoiding a large amount of manual work.

This example will be used as a running example in the remainder of this paper.

In the next section, we discuss our holistic integration proposal to the existing work of literature. The remainder of this paper is organized as follows. In Section II, we study some relevant related works. In Section III, we present our holistic integration approach applied on SOD graphs. Section IV will be devoted to comparative experiments according to other approaches. We conclude this work in Section V.

\section{RELATED WORKS}

Schema matching problem is among the most studied problems as it represents a key task in several application fields including data integration. The schema matching task consists of identifying semantic correspondences (mappings or alignments) between data models such as database schemas, XML schemas and ontologies [7]. The general workflow for schema matching [7] is composed of a pre-processing step, an execution step for one or several matchers, a combination of

\footnotetext{
${ }^{2}$ http://data.worldbank.org/indicator

${ }^{3}$ http://data.worldbank.org/country/united-kingdom

${ }^{4}$ https://www.gov.uk/government/statistical-data-sets/structure-of-theagricultural-industry-in-england-and-the-uk-at-june
} 
matcher(s) results and finally a selection of correspondences. We have observed that one or several steps in this workflow are reduced to a combinatorial optimisation problem. The latter criteria and further ones have been summarized in Table I to compare our approach, named LP4HM, with some related works. The criteria we have chosen are: (1) the type of approaches, namely pairwise or holistic, (2) the internal model representation, (3) the application to a domain, (4) the reduction to a known combinatorial optimisation problem, its complexity and in which step the reduction is noticed, (5) the dependence of a threshold and (6) the elemental or structural techniques. We refer to [8] for the classification of these techniques. We will first describe some related works, then we will discuss the main differences and similarities of our approach compared to these works.

Holistic Approaches: These approaches generate correspondences for several input schemas. In this paragraph, we describe DCM [9], HSM [10], PORSCHE [11] and PLASMA [12] holistic propositions. DCM and HSM are applied for matching web query interfaces. Their schemas are a list of attributes extracted from the web query interfaces. The Dual Correlation Mining (DCM) algorithm computes groups of positively correlated attributes (co-occur in the same query interface). Then it discovers matching by computing negative correlation (not co-occur in the same query interface) among groups of attributes. The selection of the final correspondences is done by a greedy selection using threshold, ranking and scoring functions. The Holistic Schema Matching (HSM) is similar to the DCM approach according to the co-occurence idea. The HSM algorithm computes between every two attributes of all the schemas: (i) a matching score for the attributes that are frequently co-present and (ii) a grouping score for the attributes that are rarely co-present. The selection of the final correspondences is done by an iterative algorithm using grouping scores and thresholds. PORSCHE and PLASMA holistic approaches handle tree XML or XSD schemas. PORSCHE focuses on Book domain and PLASMA on E-Business domain. Both approaches use the clustering technique. PORSCHE uses the tree mining technique in order to construct clusters of similar elements. Element-level similarity is based on a local thesaurus and abbreviation. An incremental algorithm is applied to compute final correspondences using node ranks representing the node contexts. PLASMA applies, holistically, the algorithm Dryade [13] to extract the frequent sub-trees, then they compute string-based similarities between the elements of these sub-trees in order to keep the most relevant ones. To compute structural similarities, they apply an enhanced version of the EXSMAL [14] algorithm between all pairs of frequent subtrees independently. Different thresholds and weighting are used in PLASMA to combine and select correspondences.

Pairwise Approaches: These approaches generate correspondences for two input schemas. In this paragraph, we describe COMA++ [15], Similarity Flooding [16], BMatch [17], CODI [18] and OLA [19] pairwise approaches. COMA++ is a generic pairwise matcher applied for ontologies, XML schema and relational databases. It transforms input sources into a directed acyclic graph. Seventeen elemental and structural level matchers are executed in parallel. The structural matchers focus on computing structural similarities between children, paths and leaves. The Similarity Flooding (SF) pairwise approach converts input sources into labelled graphs. The structural-level matcher of SF takes as input the similarities of string-based element level matchers, then it propagates similarities between neighbourhood nodes until a fixed point computation. One among the filters of SF is based on the stable marriage problem that returns a local optimal solution [20]. The BMatch [17] pairwise approach handles tree schemas. It combines two element-level matchers and a structural-level matcher. This latter measures structural similarities based on the context of nodes and uses a b-tree to improve the matching performance.

The pairwise matcher CODI implements the probabilistic Markov logical framework presented in [21] for ontology matching. This framework transforms the matching problem to a maximum-a-posteriori (MAP) optimisation problem that is equivalent to Max-Sat problem (NP-hard). The MAP problem encompasses cardinality and coherence constraints, as well as stability constraints. It aims to maximize the probability of the potential alignments. This framework is generic since it considers classes, properties and individuals in the ontologies. It is solved through an Integer Linear Program (ILP) but the authors of [21] omit the details of their program. The pairwise approach OLA aims to match ontologies. It uses labelled graphs as internal representation. The resolution of this problem is carried out by an iterative algorithm. Indeed, the OLA algorithm takes as input the similarities resulting from the element-level matcher, then computes iteratively structural similarities for properties and elements. It does it through a set of equations. The resolution finishes when a fixed point is attempted. The filtering step of OLA is reduced to a weighted bipartite graph matching problem.

Discussion: Unlike the other approaches, only CODI and LP4HM can resolve, in the same time, the structural matching phase without additional structural similarity computation and the correspondences extraction phase. All the other approaches compute an additional structural similarity through their structural-level matcher. The integer linear program of LP4HM is reduced, in pairwise scenarios, to the maximum weight bipartite graph matching problem (MWBG), just like what OLA has done for the filtering phase. The complexity of the maximum-weighted bipartite graph matching problem with an integer linear program is polynomial [22], even with the simplex algorithm [22] [23]. For holistic scenarios, LP4HM is reduced to the maximum weighted non-bipartite graph matching problem [22] which can also be solved in polynomial time by some algorithm like the Edmonds' algorithm [24]. Unlike CODI, whose pairwise approach is reduced to an NPHard problem, our proposed solution extends a polynomial problem in both pairwise and holistic versions.

Another point of discussion is the optimality of the proposed solution. In combinatorial optimisation, we distinguish local and global optimal solutions. A local optimum is a solution better than all neighbourhood solutions whilst a global optimum is the best solution among all the possible solutions of a problem. In the same conditions, local optimum is lower than or equal to global optimum. For the pairwise matching approaches SF and OLA, the authors of [20] emphasizes that the stable marriage filter of SF returns a local optimal solution and the maximum weight matching filter of OLA returns a global optimal solution. They also show through the example 
TABLE I. A SYNTHETIC COMPARISON OF LP4HM WITH SOME RELATED WORKS

\begin{tabular}{|c|c|c|c|c|c|c|c|c|c|}
\hline \multirow[t]{2}{*}{ Approach } & \multirow[t]{2}{*}{ Type } & \multirow{2}{*}{$\begin{array}{l}\text { Internal } \\
\text { Model }\end{array}$} & \multirow{2}{*}{$\begin{array}{l}\text { Domain } \\
\text { App. }\end{array}$} & \multicolumn{3}{|c|}{ Combinatorial Optimisation Reduction } & \multirow{2}{*}{$\begin{array}{l}\text { Dep. } \\
\text { Thresh } \\
\text { old }\end{array}$} & \multicolumn{2}{|c|}{ Matching techniques } \\
\hline & & & & Problem & Complexity & Used In & & Element-level & Structural-level \\
\hline DCM & holistic & $\begin{array}{l}\text { list of at- } \\
\text { tributes }\end{array}$ & Yes & - & - & - & Yes & linguistic based & - \\
\hline HSM & holistic & $\begin{array}{l}\text { list of at- } \\
\text { tributes }\end{array}$ & Yes & - & - & - & Yes & linguistic based & - \\
\hline PORSCHE & holistic & tree & Yes & - & - & - & No & linguistic-based & tree mining \\
\hline $\begin{array}{l}\text { Similarity } \\
\text { Flooding }\end{array}$ & pairwise & $\begin{array}{l}\text { labelled } \\
\text { graphs }\end{array}$ & No & $\begin{array}{l}\text { Stable } \\
\text { Marriage }\end{array}$ & polynomial & filtering & Yes & string-based & $\begin{array}{l}\text { structural } \\
\text { similarity } \\
\text { propagation } \\
\text { until fixed point } \\
\text { computation } \\
\end{array}$ \\
\hline OLA & pairwise & $\begin{array}{l}\text { labelled } \\
\text { graph }\end{array}$ & No & $\begin{array}{l}\text { Maximum } \\
\text { weighted } \\
\text { graph } \\
\text { matching }\end{array}$ & polynomial & filtering & Yes & $\begin{array}{l}\text { string constraint, } \\
\text { linguistic based }\end{array}$ & $\begin{array}{l}\text { similarity } \\
\text { equation fixed } \\
\text { point }\end{array}$ \\
\hline LP4HM & holistic & $\begin{array}{l}\text { directed } \\
\text { acyclic } \\
\text { graphs }\end{array}$ & No & $\begin{array}{l}\text { Maximum } \\
\text { weighted } \\
\text { graph } \\
\text { matching }\end{array}$ & polynomial & $\begin{array}{l}\text { structural } \\
\text { matcher, } \\
\text { filtering }\end{array}$ & No & $\begin{array}{l}\text { string and lin- } \\
\text { guistic based }\end{array}$ & $\begin{array}{l}\text { integer linear con- } \\
\text { straints }\end{array}$ \\
\hline
\end{tabular}

on pages 132-136 ([20]) that the local optimum of SF is lower than the global optimum of OLA for pairwise matching in the same conditions. Since our approach is reducible to finding a maximum weight matching (in bipartite graph in case of pairwise matching or in non-bipartite graph in case of holistic matching), our approach returns also globally optimal solutions representing the best assignments over all possible solutions maximizing the sum of the weights.

We note some differences between OLA and our approach: OLA uses an iterative algorithm to compute structural similarities then it applies the principle of the MWBG in filtering; LP4HM extends the integer linear program of the MWBG with structural constraints without additional structural similarity computation. OLA focuses on ontologies while LP4HM focuses on hierarchical trees to resolve SOD integration problem in multidimensional data warehouses. LP4HM can be applied for taxonomic ontologies but not for the other types of ontologies.

The use of the holistic approaches DCM and HSM is limited to a list of attributes, so they are not applicable on structured schemas namely hierarchical graphs. The holistic approaches PORSCHE and PLASMA are relevant for the context of our study but, as far as we know, there are no available tools to make comparison. PORSCHE is based in an incremental algorithm and PLASMA computes independent matching problems between common sub-trees. So both PORSCHE and PLASMA do not explore all possible solutions which leads to suppose that their solutions are locally optimal.

\section{Holistic Statistical Open Data Integration}

This section is devoted to present our holistic integration approach based on the integer linear programming (ILP) technique.

\section{A. An Example of Input Graphs}

The input graphs of the holistic integration step are the results of the "extraction and annotation" step, as mentioned in section I-A. To help users better understand the content of these graphs, we present: (i) in Fig.2(a) an excerpt of three spreadsheets belonging to our motivating example, and (ii) in Fig.2(b) an excerpt of these spreadsheets transformed into graphs.

Fig.2(a) depicts three SOD spreadsheets "A", "B" and "C" available in the link ${ }^{5}$. "A" shows annual cereal yields in UK, "B" shows annual wheat (a cereal type) production, yield and area per region and per year. "C" shows crops production per year in England. These tables are composed of numerical blocks containing statistical data indexed by a StubHead (row header) and BoxHead (column header). The StubHead and BoxHead form a part of the structural data that we will use in the integration step.

In Fig.2(a), we observe that the StubHead of "A", the BoxHead of "B" and the BoxHead of "C" depict structural data on year. The BoxHead of "A" and the StubHead of "C" show structural data on cereal types. The spreadsheet "B" presents details on wheat existing in sources "A" and "C". Our aim

\footnotetext{
${ }^{5}$ https://www.gov.uk/government/statistical-data-sets/structure-of-theagricultural-industry-in-england-and-the-uk-at-june
} 
is to integrate these sources to get a better view of these data. Hence, we have transformed these sources into graphs as depicted in Fig.2(b) $G_{1}, G_{2}, G_{3}$, corresponding respectively to the sources "A", "B", "C".

Each directed acyclic graph, denoted as $G_{i}=\left(V_{i}, E_{i}\right)$, is composed of:

- $\quad$ Two types of vertices $V_{i}$ : structural vertices $V_{i}^{S}$ and numerical vertices $V_{i}^{N}$. In the integration step, we use only structural vertices as they represent the schema of the tables. To simplify, we note $V_{i}^{S}$ as $V_{i} . V_{i}=$ $\left\{v_{i_{k}}, \forall k \in\left[1,\left|V_{i}\right|\right]\right\}$ where $k$ refers to the vertex order in the graph according to a Depth-First Search (DFS) algorithm applied on $V_{i}$.

- Two types of edges $E_{i}$ : the $E_{i}^{S S}$ edges are defined between two different structural vertices and the $E_{i}^{S N}$ edges are defined between structural and numerical vertices. Only the first type of edges will be involved in the integration step. To simplify, we note $E_{i}^{S S}$ as $E_{i} . E_{i}=\left\{e_{i_{k, l}}=\left(v_{i_{k}}, v_{i_{l}}\right), \forall k, l \in\left[1,\left|V_{i}\right|\right]\right\}$. The latter result from the hierarchical concept classification between structural vertices; for more details on algorithms readers can refer to [1]. For instance, $G_{1}$ is composed of two hierarchies. The first one represents cereal types that we can observe on the flattened BoxHead of the source "A". The second one represents years corresponding to the flattened StubHead of the source "A".

Notations: We summarize the notations defined above and others that will be used in the remainder of this paper:

- $\quad N$ is the number of input graphs.

- $\quad i, j$ are graph numbers used for $G_{i}$ and $G_{j}$.

- $V_{i}$ is the set of structural vertices in the graph $G_{i}$.

- $n_{i}=\left|V_{i}\right|$ is the order of the graph $G_{i}$.

- $E_{i}$ is the set of structural edges in the graph $G_{i}$.

- $i_{k}$ is the index of the vertex of order $k$ in the graph $G_{i}$.

- $j_{l}$ is the index of the vertex of order $l$ in the graph $G_{j}$.

- $i_{\text {pred(k) }}$ is the index of the predecessor of the vertex $v_{i_{k}}$. (we have at most one predecessor per vertex as we have strict-hierarchies [1] [6] and directed acyclic graphs).

\section{B. Pre-Matching Phase}

The pre-matching phase aims to prepare the input data of the matching process. It takes a set of $N$ directed acyclic graphs $G_{i}=\left(V_{i}, E_{i}\right) i \in[1, N], N \geq 2$ representing only structural schema elements. It produces: (1) $\sum_{i=1}^{N-1}(N-i)$ similarity matrices representing the result of element-level matchers and (2) $N$ direction matrices computed independently representing the hierarchical relationships between structural vertices.
1) Similarity Matrices: We compute $\sum_{i=1}^{N-1}(N-i)$ similarity matrices denoted $\operatorname{Sim}_{i, j}$ of size $n_{i} \times n_{j}$ defined between two different graphs $G_{i}$ and $G_{j}, \forall i \in[1, N-1], j \in[i+1, N]$. Each matrix encodes similarity measures. The similarity measures are computed on the labels of vertices. These labels are first tokenized and stemmed before four types of elementlevel matchers are applied. We have chosen the maximum as an aggregation function between the element-level matchers. Two string-based matchers and two linguistic-based matchers are used. The string-based matchers compute the Jaccard and cosine (with Term Frequency Inverse Document Frequency "TF.IDF" for the weights of vectors of each token) distances. The linguistic-based matchers compute the Wup [25] and Lin [26] distances using the thesaurus Wordnet. For each pairwise graph $G_{i}$ and $G_{j}$, the similarity measure is computed between all combination of pairwise nodes $v_{i_{k}}$ and $v_{j_{l}}$ belonging respectively to $G_{i}$ and $G_{j}$. The similarity measure is defined as follows:

$$
\begin{gathered}
\operatorname{sim}_{i_{k}, j_{l}}=\max _{\left(\operatorname{Jacc}\left(v_{i_{k}}, v_{j_{l}}\right), \operatorname{Cosine}\left(v_{i_{k}}, v_{j_{l}}\right)\right.} \\
\left.\operatorname{Wup}\left(v_{i_{k}}, v_{j_{l}}\right), \operatorname{Lin}\left(v_{i_{k}}, v_{j_{l}}\right)\right)
\end{gathered}
$$

Hence, each similarity matrix is defined as follows:

$$
\operatorname{Sim}_{i, j}=\left\{\operatorname{sim}_{i_{k}, j_{l}}, \forall k \in\left[1, n_{i}\right], \forall l \in\left[1, n_{j}\right]\right\}
$$

2) Direction Matrices: We compute a set of $N$ direction matrices $D i r_{i}$ of size $n_{i} \times n_{i}$ defined for each graph $G_{i}, \forall i \in$ $[1, N]$. Each matrix encodes edges' direction and is defined as follows:

$$
\begin{aligned}
\operatorname{Dir}_{i}=\left\{\operatorname{dir}_{i_{k, l}},\right. & \left.\forall k \times l \in\left[1, n_{i}\right] \times\left[1, n_{i}\right]\right\} \\
& \operatorname{dir}_{i_{k, l}}= \begin{cases}1 & \text { if } e_{i_{k, l}} \in E_{i} \\
-1 & \text { if } e_{i_{l, k}} \in E_{i} \\
0 & \text { otherwise }\end{cases}
\end{aligned}
$$

\section{A Linear Program For Holistic Matching (LP4HM)}

In this section, we present a 0-1 linear program to automatically solve the SOD graph matching problem. It maximizes a linear objective function over a finite number of binary variables (encoding matching vertices) subject to a set of inequalities defined according to decision variables. It proposes a holistic solution by dealing with $\sum_{i=1}^{N}(N-i)$ combination of pairwise graphs among the $N$ input graphs (we reduce the size of input combinations by working in an upper triangular matrix representing the combinations of all pairwise input graphs).

1) Decision Variables: Our model includes a single decision variable. It exhibits the possibility to have or not have a matching between two vertices belonging to two different input graphs.

For each $G_{i}$ and $G_{j}, \forall i \in[1, N-1], j \in[i+1, N], x_{i_{k}, j_{l}}$ is a binary decision variable equal to 1 if the vertex $v_{i_{k}}$ in the graph $G_{i}$ matches with the vertex $v_{j_{l}}$ in the graph $G_{j}$ and 0 otherwise. Fig.2(b) shows some decision variables between vertices, for instance $x_{1_{8}, 3_{10}}$ is defined between the vertices $v_{1_{8}}$ in $G_{1}$ and $v_{3_{10}}$ in $G_{3}$. 


\begin{tabular}{|r|r|rr|}
\hline A & Wheat & \multicolumn{1}{|c|}{ Barley } & \multicolumn{1}{l|}{ Oats } \\
\hline 2004 & 7,8 & 5,8 & 5,8 \\
2005 & 8,0 & 5,9 & 5,8 \\
2006 & 8,0 & 5,9 & 6,0 \\
2007 & 7,2 & 5,7 & 5,5 \\
2008 & 8,3 & 6,0 & 5,8 \\
\hline
\end{tabular}
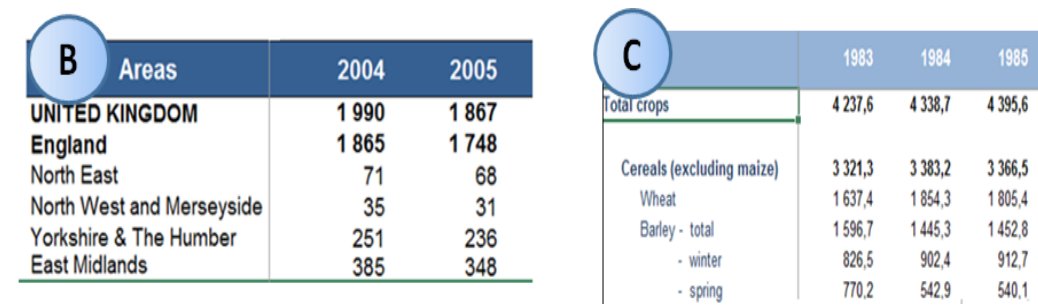

(a) Open Data input spreadsheets

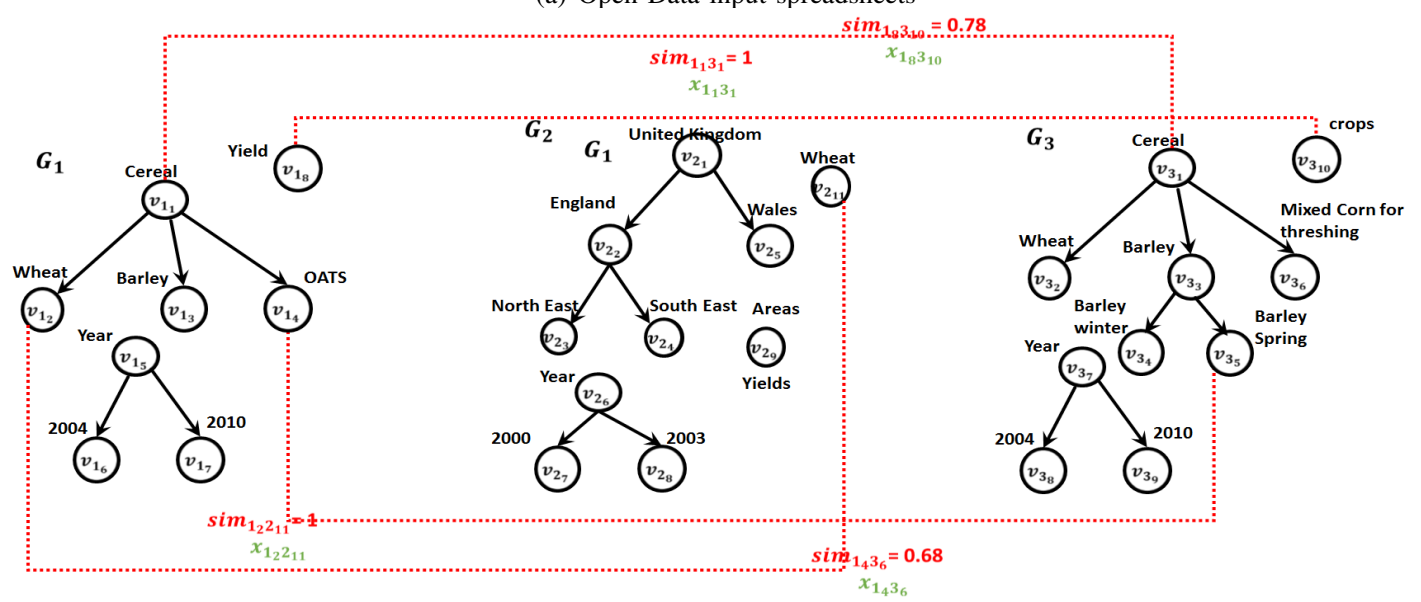

(b) Open Data transformed into Graphs

Fig. 2. An example of Open Data Input Graphs.

2) Linear Constraints: Linear constraints are generally equivalent to logical implications between decision variables. The theorem 1 of [27] shows the relation between logical implications and linear inequalities. Our model constraints have been modelled by following this theorem.

Theorem 1. Let $x_{i}$ be a $0-1$ variable for all $i$ in some finite set $I$ and $y$ be a $0-1$ variable:

$\left\{\right.$ If $x_{i}=0$ for all $i \in I$ Then $\left.y=0\right\} \Longleftrightarrow\left\{y \leq \sum_{i \in I} x_{i}\right\}$.

Our model includes two types of constraints: (i) Matching Setup (MS) constraints and (ii) Graph Structure (GS) constraints.

The former belongs to matching setup. MS1 encodes the 1:1 matching cardinality [2]. MS2 encodes constraints to select correspondences with similarity greater than a given threshold. We emphasize that the default version of our matcher runs without the MS2 constraint. However, we are aware that some expert users may require specific threshold values. So, we offer the possibility to perform our matcher with a given threshold.

MS1 (Matching Cardinality) Each vertex $v_{i_{k}}$ in the graph $G_{i}$ could match with at most one vertex $v_{j_{l}}$ in the graph $G_{j}, \forall i \in[1, N-1], j \in[i+1, N]$.

$$
\sum_{l=1}^{n_{j}} x_{i_{k}, j_{l}} \leq 1, \quad \forall k \in\left[1, n_{i}\right]
$$

Example 1. Considering the vertex $v_{1_{1}}$, two constraints are generated by applying MS1 :

$$
\begin{aligned}
& \sum_{l=1}^{11} x_{1_{1}, 2_{l}} \leq 1 \\
& \sum_{l=1}^{10} x_{1_{1}, 3 l} \leq 1
\end{aligned}
$$

Bearing in mind that we have binary decision variables $(\in\{0,1\})$, at most one decision variable in the inequalities (1) and (2) can be checked (equals to 1). Otherwise, if all decision variables in (1) and (2) are still unchecked (equals to 0 ), no correspondences will be found for the vertex $v_{1_{1}}$.

MS2 (Matching Threshold) For a given thresh, our model encodes the threshold setup in the following constraint: $\forall i \in[1, N-1], j \in[i+1, N]$ and $\forall k \times l \in$ $\left[1, n_{i}\right] \times\left[1, n_{j}\right]$

$$
\operatorname{sim}_{i_{k}, j_{l}} x_{i_{k}, j_{l}} \geq \operatorname{thresh} x_{i_{k}, j_{l}}
$$

Example 2. Given the two vertices $v_{1_{8}}$ (yield) in $G_{1}$ and $v_{3_{10}}$ (crops) in $G_{3}$, and a thresh inputted by users, the constraint generated for MS2 is as follows:

$$
\operatorname{sim}_{1_{8}, 3_{10}} x_{1_{8}, 3_{10}} \geq \text { thresh } x_{1_{8}, 3_{10}}
$$

○ If thresh $=0.6$ and $\operatorname{sim}_{1_{8}, 3_{10}}=0.78$

Then $0.78 x_{1_{8}, 3_{10}} \geq 0.6 x_{1_{8}, 3_{10}}$, constraint (3) is satisfied regardless the value of $x_{1_{8}, 3_{10}}$.

- If $x_{1_{8}, 3_{10}}=1$ vertices are matched and their similarity is superior than the thresh.

- If $x_{1_{8}, 3_{10}}=0$ vertices are not matched because they are not part of the global optimum.

○ If thresh $=0.8$ and $\operatorname{sim}_{1_{8}, 3_{10}}=0.78$

Then $0.78 x_{1_{8}, 3_{10}} \geq 0.8 x_{1_{8}, 3_{10}}$, to satisfy the constraint (3), $x_{1_{8}, 3_{10}}$ must be equal to 0 i.e no matching vertices if their similarity is inferior than the thresh. 


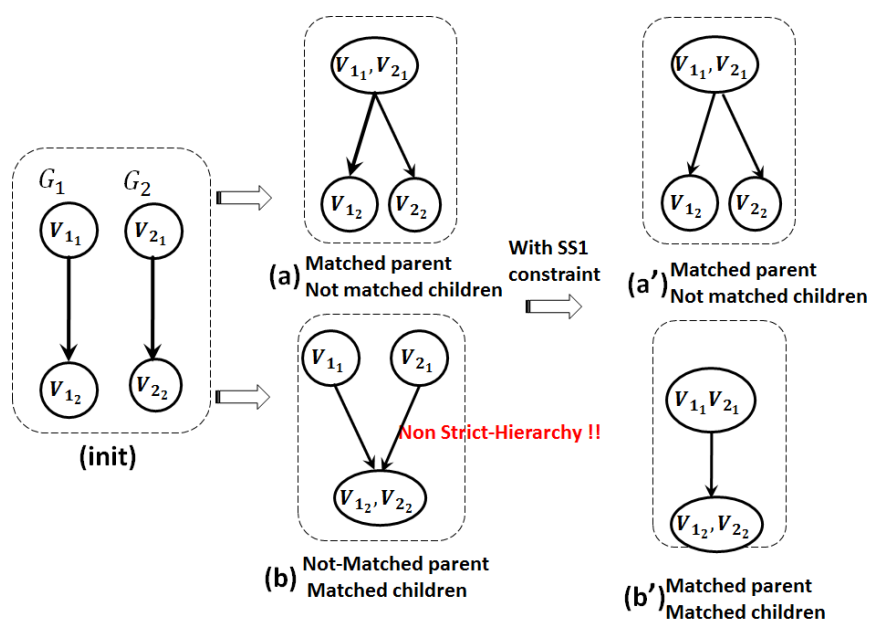

Fig. 3. Example of Strict Hierarchy Constraint Impact

The second type of constraints represents the main original parts of our model: (i) they will prepare the generation of a hierarchically integrated graph by avoiding different edges directions which accelerates and facilitates the identification of the hierarchies and dimensions of the multidimensional schemas and (ii) they will anticipate the resolution of the nonstrict hierarchy problem which gives rise to summarizability problems [6] in data analysis. For instance, suppose that we want to analyse the number of sales for each type of product in a multidimensional schema. This schema has a non strict hierarchy "type of product $\rightarrow$ product" i.e an instance of a product belongs to different types of products. So, this instance causes a double counting problem for sales due to the nonstrictness of the hierarchy.

In the following, we propose the SS1 constraint to generate strict hierarchies and the SS2 constraint to generate simple edges' directions.

SS1 (Strict hierarchies) This constraint allows us to resolve the non-strict hierarchy problem [6]. Fig.3 depicts different situations; on the left side we have two simple input graphs $G_{1}$ and $G_{2}$ (init), in the center we have two different situations (a) and (b) of integrated graphs and on the right side we have the resulting situations (a') and (b') when we apply the constraint SS1. The situation (a) shows the case when parents match and children do not match; this case is not conflictual for hierarchies. The situation (b) shows the case when parents do not match and children match; this case is conflictual because it generates non-strict hierarchy (the integrated child node has two parents). When we apply the constraint SS1, we generate two non conflictual situations (a') and (b'). The constraint SS1 is as follows: $\forall i \in[1, N-1], j \in[i+1, N]$ such as $\forall k \times l \in\left[1, n_{i}\right] \times\left[1, n_{j}\right]$ :

$$
x_{i_{k}, j_{l}} \leq x_{i_{\text {pred }(k)}, j_{\text {pred }(l)}}
$$

Example 3. The SS1 constraint generated for the example of Fig. 3 is :

$x_{1_{2}, 2_{2}} \leq x_{1_{1}, 2_{1}}(4)$

$\circ$ If $x_{1_{2}, 2_{2}}=0$ Then the constraint (4) is satisfied regardless the value of $x_{1_{1}, 2_{1}}$.
- If $x_{1_{1}, 2_{1}}=0$ then we get situation (init).

- If $x_{1_{1}, 2_{1}}=1$ then we get situation (a').

- If $x_{1_{2}, 2_{2}}=1$ Then to satisfy the constraints (4), $x_{1_{1}, 2_{1}}$ must be equal to 1 , which corresponds to situation (b').

SS2 (Edge Direction) The purpose of this constraint is to prevent the generation of conflictual edges. On the left side of Fig.4, we have two input graphs $G_{1}$ and $G_{2}$, on the right side we have two sets of possible integration situations which depend on the product of edges' directions. When the product's edges' direction is equal to 1 , we notice that we have situations similar to those previously explained in the constraint SS1. When product's edges' direction is equal to 1 , we notice case (a), in which the integrated graph is no longer simple. To generate simple integrated graphs, we propose the following constraint, which will be applied when edges' direction is equal to 1: $\forall i \in[1, N-1], j \in[i+1, N]$ such as $\forall k, k^{\prime} \in$ $\left[1, n_{i}\right] \forall l, l^{\prime} \in\left[1, n_{j}\right]$

$$
x_{i_{k}, j_{l}}+x_{i_{k^{\prime}}, j_{l^{\prime}}}+\left(\operatorname{dir}_{i_{k, k^{\prime}}} d i r_{j_{l, l^{\prime}}}\right) \leq 0
$$

Example 4. The SS2 constraints generated for the example of Fig.4 is :

$$
\begin{array}{ll}
x_{1_{1}, 2_{2}}+x_{1_{2}, 2_{1}}+\left(\operatorname{dir}_{1_{1,2}} \operatorname{dir}_{2_{2,1}}\right) & \leq 0 \\
x_{1_{1}, 2_{1}}+x_{1_{2}, 2_{2}}+\left(\operatorname{dir}_{1_{1,2}} \operatorname{dir}_{2_{1,2}}\right) & \leq 0
\end{array}
$$

- If $x_{1_{1}, 2_{2}}=1$ and $x_{1_{2}, 2_{1}}=1$, knowing that we have $d i r_{1_{1,2}}=-1$ and $d i r_{2_{2,1}}=-1$ Then constraint (5) is not satisfied $(1 \leq 0)$. This case corresponds to situation (a) in Fig.4.

3) Resulting Model: The objective of our model is to maximize the sum of the similarities between matched vertices. Using the constraints mentioned above, our resulting model is as follows:

$$
\begin{aligned}
& \int \max \sum_{i=1}^{N-1} \sum_{j=i+1}^{N} \sum_{k=1}^{n_{i}} \sum_{l=1}^{n_{j}} \operatorname{sim}_{i_{k}, j_{l}} x_{i_{k}, j_{l}} \\
& \text { s.t. } \quad \sum_{l=1}^{n_{j}} x_{i_{k}, j_{l}} \leq 1, \forall k \in\left[1, n_{i}\right] \\
& \forall i \in[1, N-1], j \in[i+1, N] \\
& \operatorname{sim}_{i_{k}, j_{l}} x_{i_{k}, j_{l}} \geq \operatorname{thresh} x_{i_{k}, j_{l}} \\
& \forall i \in[1, N-1], j \in[i+1, N] \\
& \forall k \in\left[1, n_{i}\right], \forall l \in\left[1, n_{j}\right] \\
& x_{i_{k}, j_{l}} \leq x_{i_{\text {pred }(k),}, j_{\text {pred }(l)}} \\
& \forall i \in[1, N-1], j \in[i+1, N] \\
& \forall k \in\left[1, n_{i}\right], \forall l \in\left[1, n_{j}\right] \\
& x_{i_{k}, j_{l}}+x_{i_{k^{\prime}}, j_{l^{\prime}}}-\left(\operatorname{dir}_{i_{k, k^{\prime}}} d i r_{j_{l, l^{\prime}}}\right) \leq 1 \\
& \forall i \in[1, N-1], j \in[i+1, N] \\
& \forall k, k^{\prime} \in\left[1, n_{i}\right], \forall l, l^{\prime} \in\left[1, n_{j}\right] \\
& x_{i_{k}, j_{l}} \in\{0,1\} \quad \forall i \in[1, N-1], j \in[i+1, N]
\end{aligned}
$$

Our model has: 


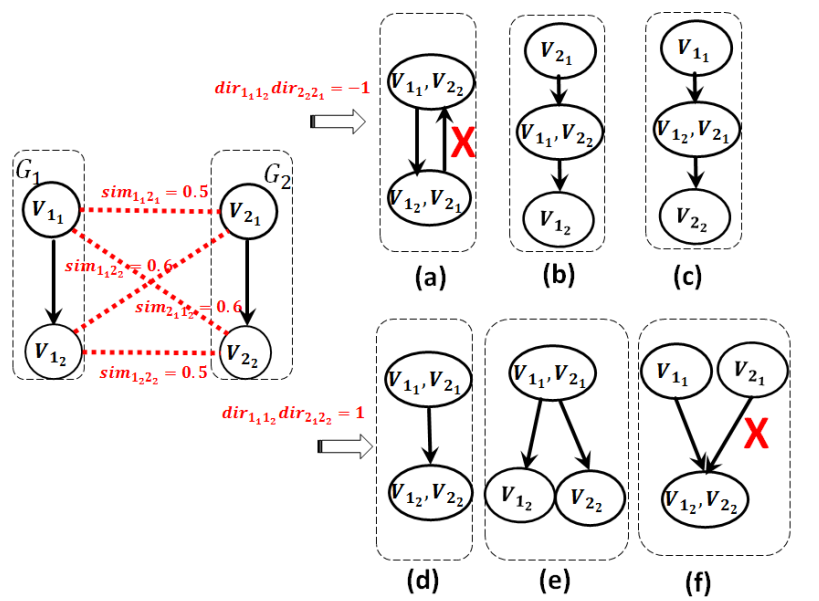

Fig. 4. Example of Edge Direction Constraint Impact

- $\quad \sum_{i=1}^{N-1} \sum_{j=i+1}^{N} n_{i} n_{j}$ decision variables

- $\quad \sum_{i=1}^{N} n_{i}(N-i)$ constraints of type MS1.

- The constraints MS2 are $\sum_{i=1}^{N} n_{i}-1$.

- The constraints SS1 are $\leq \sum_{i=1}^{N-1} n_{i}-1$.

- The constraints SS2 are $\leq \sum_{i=1}^{N-1} \sum_{j=i+1}^{N}\left|E_{i}\right|\left|E_{j}\right|$.

The LP4HM program focuses on 1:1 matching cardinalities using $0-1$ decision variables. We propose to relax the decision variables in the $[0,1]$ interval. This relaxation enables resolving $\mathrm{n}$ :m matching cardinalities. Suppose that we have two vertices "first name" and "last name" both having the same similarity distance to "name". Therefore, we have two 0-1 decision variables with the same similarity factor, only one of these decision variables will be chosen. By relaxing variables in the $[0,1]$ interval both variables will be assigned with a 0.5 value. We named LP4HM(relax) a relaxed version of LP4HM with decision variables in $[0,1]$ resolving complex matching cardinalities (n:m).

In the related work section, we have shown that almost all approaches require tuning threshold to improve their matching quality. We think that searching a global optimal solution can help us to overcome this problem. Hence, we propose that our default LP4HM runs without the constraint MS2. These propositions will be studied experimentally in the next section.

\section{Evaluation}

In this section, we present three types of evaluations conducted on the LP4HM program. In the first evaluation, we assess the pairwise matching quality of our approach compared to COMA++ [15], BMatch [17] and Similarity Flooding [16]. In the second evaluation, we provide an estimation of the global optimal solution compared to a generalisation of local optimal solutions in the case of holistic matching. In the last evaluation, we test the performance of our approach according to running time resolution on several input SOD graphs. Experimentations have been carried out on a Dell PC (windows 8, Intel(R) Core i5, 2,30 Ghz processor, 8 Go RAM) and resolved by the Academic CPLEX solver.

\section{A. Matching Quality}

Given the absence of holistic tools adapted for hierarchical schemas, we have chosen to compare the matching quality of LP4HM with some pairwise approaches. The selected approaches are COMA++, BMatch and Similarity Flooding (SF). All of them handle hierarchical schemas. The comparative study is conducted on the user-oriented benchmark ${ }^{6}$ proposed by [16]. The benchmark is composed of nine pairwise matching tasks. Tasks $1,2,3,4,5$ and 6 are XML and XSD schemas and tasks 7, 8, 9 are relational database schemas. Seven users from the Stanford Database Group have proposed correspondences of cardinality 0:n for each of these tasks.

We have experimented on this benchmark: (i) our approach LP4HM without the threshold constraint; (ii) our relaxed approach LP4HM(Relax) without the threshold constraint; (iii) COMA++ by combining all the proposed strategies except the reuse and fragmentation ones; (iv) the default version of BMatch and (v) the $\mathrm{SF}^{7}$ algorithm implemented in the $\mathrm{RONDO}^{8}$ tool with a threshold equals to 1 as its authors recommended it [16]. We highlight that even if the recommended threshold in SF seems evident in this benchmark, it is not generalizable and efficient for other tasks, for instance, the results of SF on the benchmark proposed by [28]. Moreover, we point out that we cannot compare local and global optimum in this benchmark, especially between SF and our approach. For doing that, the approaches should be subject to the same input sets and the same conditions, which is not the case here. Indeed, we use different seed element-level matchers so different input sets. Moreover, SF uses a threshold and LP4HM does not use a threshold so the conditions are different too.

The matching quality is evaluated by two classes of measure. The first one is composed by precision, recall and $\mathrm{f}$ measure, which are the classical measures of the Information Retrieval domain. Precision is defined as the ratio of correct correspondences to the total number of correspondences retrieved by the system. The recall is defined as the ratio of the correct correspondences to the total number of correspondences proposed by the user. F-Measure is a harmonic mean of precision and recall. The second class is composed by accuracy [16] and HSR [28], which evaluate the post-match effort that a given user could save.

Some correlations exist between these measures. For instance, precision is correlated to accuracy. The authors of SF [16] affirm that accuracy decreases (negative results) if the precision is lower than $50 \%$. Also, recall and precision are inversely correlated, so the f-measure is considered as the compromise between them.

Table II summarizes the results of the different quality measures per average task and per average user for all approaches. The authors of SF have chosen a high threshold so their results are focused on precision, which explains their good results on accuracy (the correlated measure to precision). However, we have intentionally make the choice to experiment our approach without threshold, so it is trivial

\footnotetext{
${ }^{6} \mathrm{http}: / /$ infolab.stanford.edu/ $\sim$ melnik $/ \mathrm{mm} / \mathrm{sfa} /$

${ }^{7}$ We note that the recomputed accuracy results of SF are slightly different from those published in [16]. We think that this is due to the use of the RONDO default functions.

${ }^{8}$ http://infolab.stanford.edu/ melnik/mm/rondo/
} 
TABLE II. THE QUALITY MEASURES VALUES PER AVERAGE TASK AND PER AVERAGE USER

\begin{tabular}{|c|c|c||c|c|c|}
\hline & Precision (\%) & Recall $(\%)$ & F-Measure (\%) & Accuracy (\%) & HSR (\%) \\
\hline LP4HM & 67 & 58 & 62 & 30 & $\mathbf{8 1}$ \\
\hline LP4HM(relax) & 58 & $\mathbf{6 6}$ & 60 & 23 & $\mathbf{8 1}$ \\
\hline COMA++ & 72 & 50 & 58 & 32 & 76 \\
\hline BMatch & 22 & 47 & 28 & 0 & 69 \\
\hline Similarity Flooding & $\mathbf{8 1}$ & 55 & $\mathbf{6 5}$ & $\mathbf{4 3}$ & 80 \\
\hline
\end{tabular}

that our approach will not be the best on precision. Our approach will search a global optimal solution in all the space of solutions unconditioned with a threshold, so it is likely possible that some correspondences with low similarities will be selected in the global solution so for some users and for some tasks our approach will give not correct correspondences. Even if our approach without threshold has this bias, we can observe that our approach, especially the LP4HM(Relax), is better than SF in the recall measure, which is more significant than precision for users because it is computed based on their proposed correspondences. If we examine the compromise between precision and recall given by the f-measure, we can indeed observe that our approach reaches a very close results compared to SF even without threshold. This shows that our results are competitive to $\mathrm{SF}$, even though, there are differences in the results of precision and recall.

For the post-match effort, like the authors of [28], we think that HSR is more significant than accuracy, first because it takes into account the number of elements of the schemas, second because it does not penalize the low results of precision like accuracy. Readers can simply observe that for the BMatch approach has $0 \%$ accuracy and 69\% HSR. Our results on HSR are very close to SF too, which also shows that our approach and SF have competitive results. We point out that the HSR results of our approach and SF reveal more than $80 \%$ of postmatch effort gained.

For our approach, we can observe that LP4HM(relax) version has better results on recall and lower results in precision compared to LP4HM version. Indeed, the set of found correspondences of LP4HM(relax) is larger than that of LP4HM. So for the same set of correct correspondences, the precision of LP4HM(Relax) will be lower than that of LP4HM. Moreover, if users give n:m correspondences then the set of correct correspondences of LP4HM(relax) will be larger than that of LP4HM. So the recall of LP4HM(Relax) will be greater than that of LP4HM.

In the following, we show more detailed results as depicted in Fig.5(a)-(j). We analyse results, first, along the different tasks for an average user, second, along the different users for an average task.

Fig.5(a) and Fig.5(c) depict precision and recall by task for an average user. We point out that tasks 1, 7, 8 and 9 have flat structures compared to the nested structures (depth $\geq 3$ ) of the tasks 2, 3, 4, 5 and 6 . We observe that nested tasks are more difficult than flat tasks. LP4HM and LP4HM(relax) perform medium precision results with few exceptions. For instance, precision in task 2 is high due to the use of linguistic measures. In turn, LP4HM(relax) performs good recall results for most tasks in particular for nested tasks. For tasks 7 and 8 we have lost some information, such as the datatypes, when we fitted relational database schemas into our graphs. From this our results are lower than SF results. Task 9 consists of matching 5 tables in the left schema to one table in the right schema. For this task, our matcher, COMA++ and SF, found the correct correspondences to the right schema providing precise results. However, recall results are low due to several inconsistencies and differences between the correspondences proposed by users.

Precision and recall per users for an average task are depicted respectively in Fig.5(b) and Fig.5(d). We can see in Fig.5(b) that the precision of LP4HM is better than the precision of LP4HM(relax) for all users. In turn, the recall of LP4HM(relax) is better than the recall of LP4HM for all users.

The recall of our approach is better than that of COMA++, BMatch and SF for all users. To summarize, LP4HM is more precise than LP4HM(relax) because the former focuses on 1:1 matching cardinalities and the latter focuses on n:m matching. Contrarily, for the same reason LP4HM(relax) better meets the users that have performed 0:n correspondences.

We notice in Fig.5(g) that some accuracy results of our approach are very low. This is due to precision results that are lower than 50\% [16]. In Fig.5(h), though, the accuracy results per user are close to COMA++ and SF results. Finally, for the HSR results, both LP4HM and LP4HM(relax) perform the best results in Fig.5(i) and in Fig.5(j).

We conclude that both LP4HM and LP4HM(relax) show experimentally competitive results for different users and different matching tasks compared to SF, COMA++ and BMatch approaches. LP4HM is more precise than LP4HM(relax), but the recall of LP4HM(relax) is better than the recall of LP4HM. Both approaches perform good matching of nested tasks and good results on HSR and accuracy for the post-match effort.

Searching global optimal solution is an efficient strategy since this allows our approach to face other approaches without having to use a threshold. Users seem unanimously appreciative of the effectiveness of our approach in particular for recall and HSR. The encouraging results on the nested tasks respond exactly to a major problem we try to solve, which is integrating hierarchical Open Data structures. Finally, we think that having good recall is an interesting indicator for holistic matching. Indeed, if precision is better than recall so users have to find the missing correspondences for $N$ schemas simultaneously, which is a human difficult task. So when system returns good recall and moderate precision, users have just to eliminate the not relevant mappings proposed by the matcher.

\section{B. Global Optimum and Local Optimum In holistic Matching}

In this section, we compare the global optimal solution of our approach and the sum of local optimal solutions of the SF [16] approach, in a simple case study of holistic matching. 


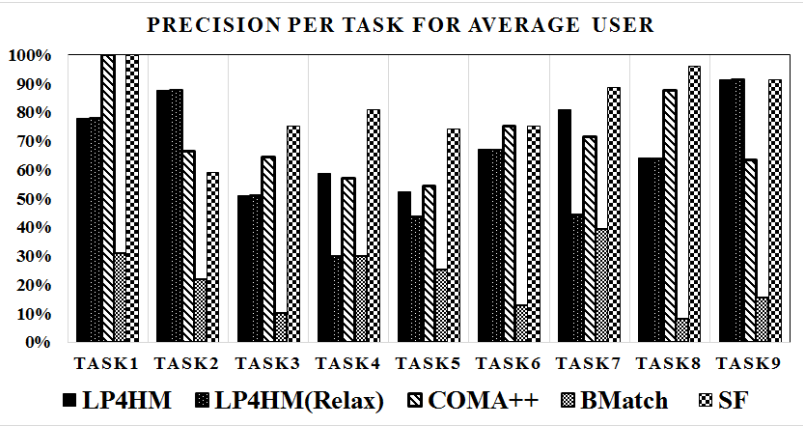

(a) Precision per task for average user RECALL PER TASK FOR AVERAGE USER

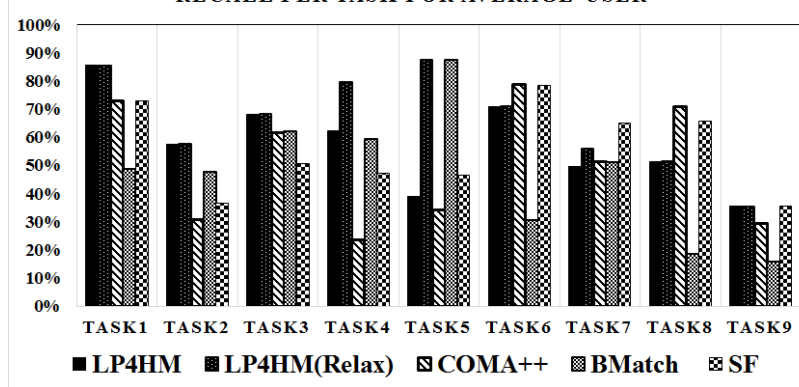

(c) Recall per task for average user F-MEASURE PER TASK FOR AVERAGE USER

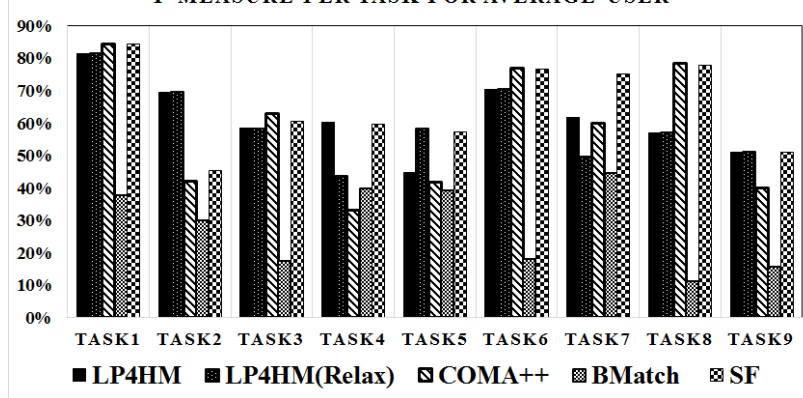

(e) F-Measure per task for average user ACCURACY PER TASK FOR AN AVERAGE USER

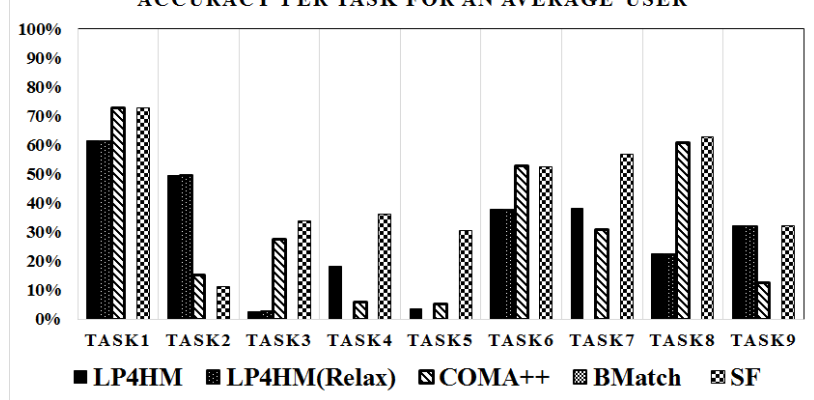

(g) Accuracy per task for average user HSR PER TASK FOR AVERAGE USER

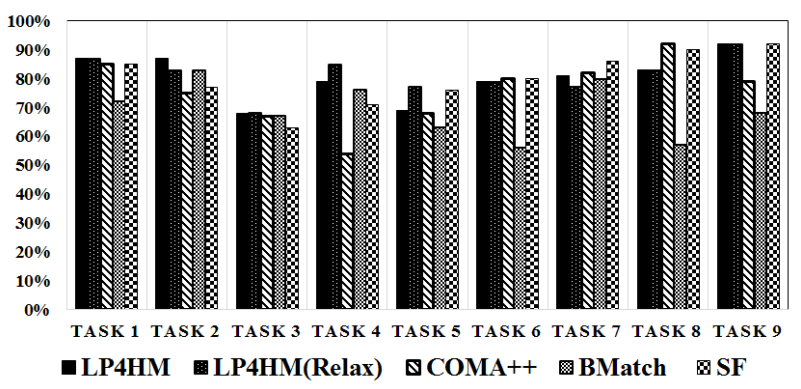

(i) HSR per task for average user

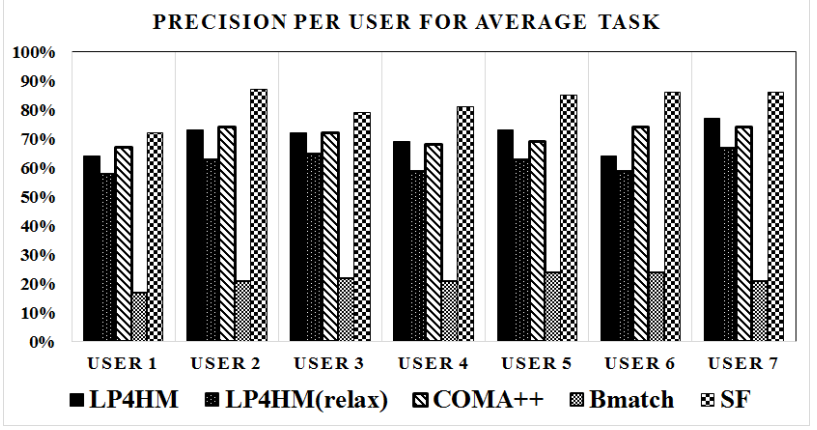

(b) Precision per user for average task RECALL PER USER FOR AVERAGE TASK

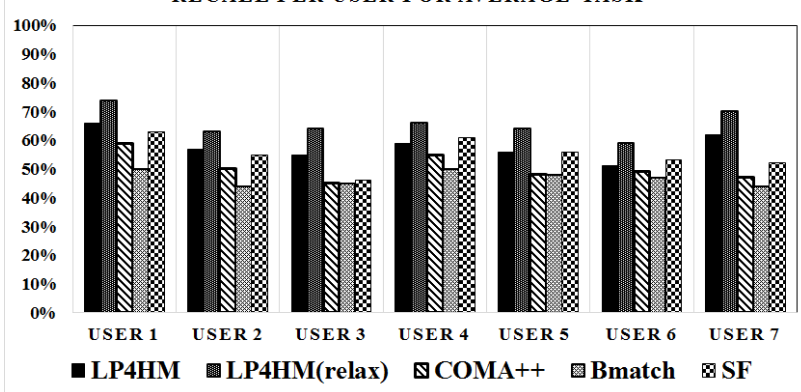

(d) Recall per user for average task

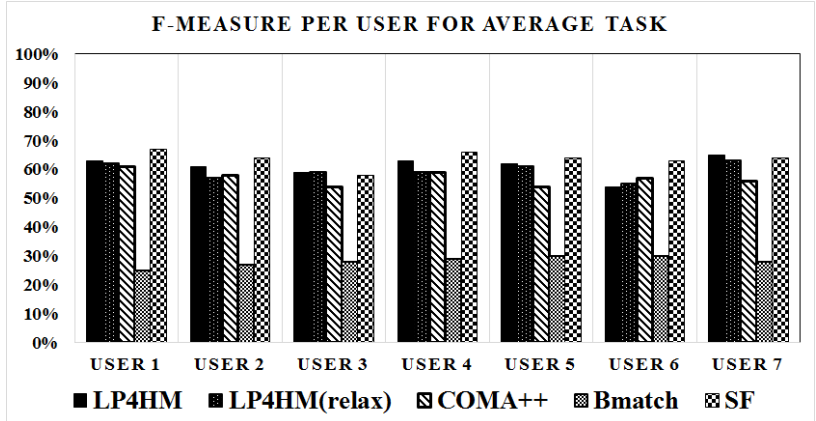

(f) F-Measure per user for average task

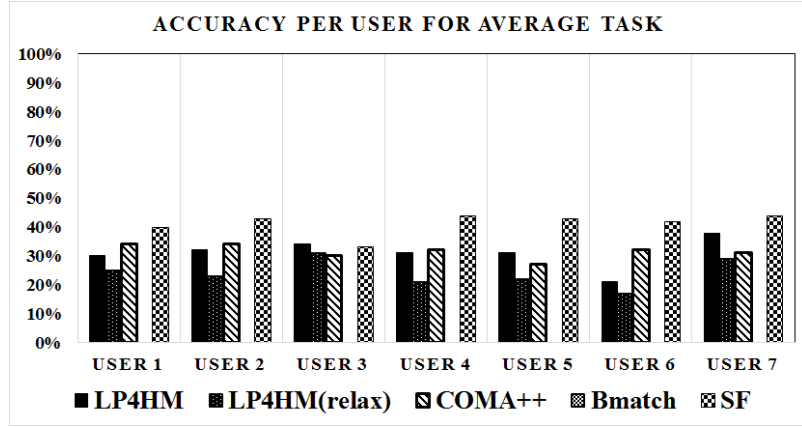

(h) Accuracy per user for average task HSR PER USER FOR AVERAGE TASK

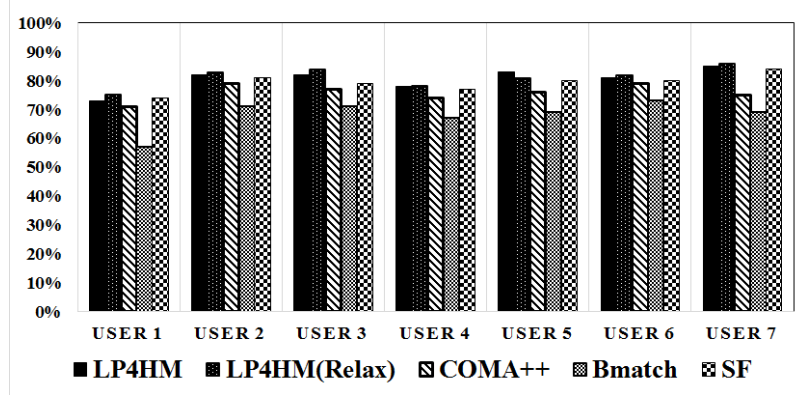

(j) HSR per user for average task

Fig. 5. Quality Measures Comparison between LP4HM, LP4HM(Relax), COMA++, BMatch, SF 
The case study consists of matching some SOD tables dealing with drugs seizure in United Kingdom. The graphs $G_{1}, G_{2}$ and $G_{3}$, in Fig.6, show an excerpt of these tables. The data of $G_{1}$ are extracted from the Scotland provider ${ }^{9}$. The data of graphs $G_{2}$ and $G_{3}$ are extracted from the UK provider ${ }^{10}$. We have computed similarities between the different nodes of the graphs (the non-specified similarities are considered as null values) and injected them to both approaches. Both approaches are used with a 0 threshold. The SF apply its propagation algorithm to compute structural similarities on the basis of injected similarities. Then, it applies its filter and extract correspondences. Our approach resolves an ILP maximizing the same input similarities under linear constraints on the structures of the graphs. The correspondences are the solutions of the ILP.
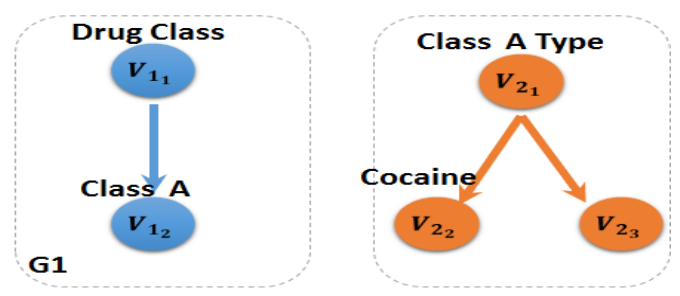

Semantic Similarities:
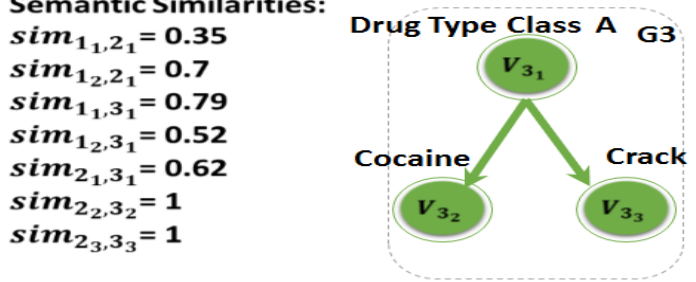

Fig. 6. A simple case study of holistic matching

The solution of the SF approach is the union of the solutions of three pairwise matching problems as follows:

$$
\begin{array}{ll}
\text { - } & \operatorname{Match}\left(G_{1}, G_{2}\right) \rightarrow\left\{\left(v_{1_{1}}, v_{2_{1}}\right)=0.35\right\} ; \\
\text { - } \quad & \operatorname{Match}\left(G_{1}, G_{3}\right) \rightarrow\left\{\left(v_{1_{1}}, v_{3_{1}}\right)=0.79\right\} ; \\
\text { - } \quad \operatorname{Match}\left(G_{2}, G_{3}\right) \rightarrow\left\{\left(v_{2_{1}}, v_{3_{1}}\right)=0.62,\left(v_{2_{2}}, v_{3_{2}}\right)=\right. \\
\left.\quad 1,\left(v_{2_{3}}, v_{3_{3}}\right)=1\right\} .
\end{array}
$$

The solution our approach LP4HM corresponds to one run taking as input $G_{1}, G_{2}$ and $G_{3}$ and returning the following correspondences:

$$
\begin{array}{ll}
\text { - } & \operatorname{Match}\left(G_{1}, G_{2}, G_{3}\right) \rightarrow \rightarrow \quad \rightarrow \quad\left\{\left(v_{1_{2}}, v_{2_{1}}\right) \quad \rightarrow \quad=\right. \\
& 0.7,\left(v_{1_{2}}, v_{3_{1}}\right)=0.52,\left(v_{2_{1}}, v_{3_{1}}\right)=0.62,\left(v_{2_{2}}, v_{3_{2}}\right)= \\
& \left.1,\left(v_{2_{3}}, v_{3_{3}}\right)=1\right\} .
\end{array}
$$

We can observe that the the sum of similarities (3.76) of the SF solution is lower than the sum of similarities (3.84) of LP4HM solution. These results support the idea that global optimal solutions represent a promising strategy for holistic schema matching problems.

\footnotetext{
${ }^{9}$ http://www.scotland.gov.uk/Topics/Statistics/Browse/CrimeJustice/TrendData

${ }^{10} \mathrm{http} / / /$ data.gov.uk/dataset/seizures-drugs-england-wales
}

\section{Matching Performance}

We evaluate the matching performance by studying the resolution time as a function of the size (number of vertices) of several input SOD graphs. Considering that our approach performs matching only on structural data, the size of each inputted graph is not very large. Indeed, in our running example for each source, structural data represents merely $12 \%$ of the total amount of information (structural and numerical data). So, the total size of inputted graphs in our experimentation is significant for our automatic integration study on SOD.

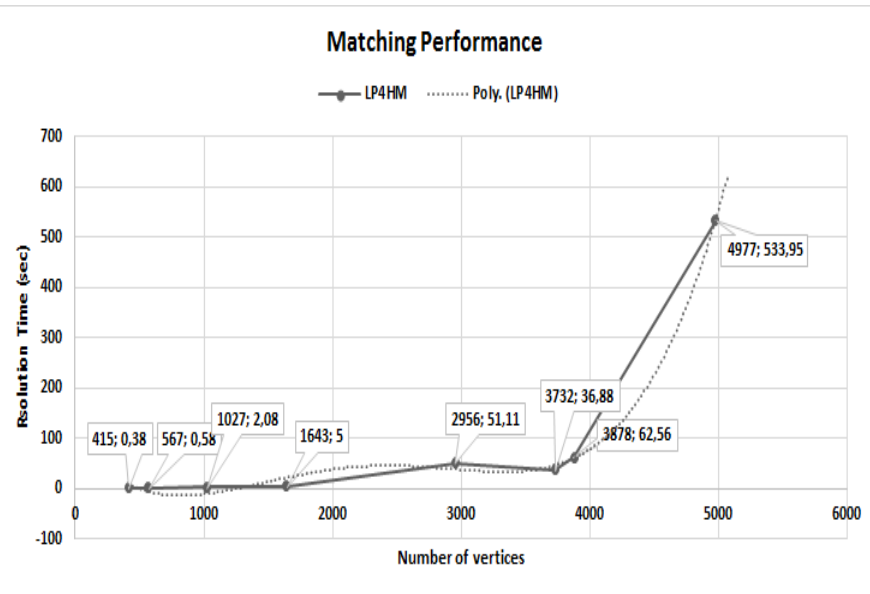

Fig. 7. Resolution time as a function of number of vertices

We have experimented LP4HM for a holistic integration of the graphs of our motivating example. We remind readers that these graphs were generated automatically from the first step of our ETL processes. The LP4HM model was solved using the Cplex solver. Fig.7 depicts the resolution time as a function of the number of vertices of several input graphs. The continuous curve connects the measured values and the discontinuous curve is a trend-line of the first one. We can observe, experimentally, that the resolution time has a polynomial trend-line $\mathrm{O}\left(n^{4}\right)$ according to the number of vertices. These results join the polynomial dissertation of [24] about the weighted graph matching problem, even though we have used a different technique. We can notice that for 415 vertices ( 9 input schemas), our model found a solution in $0,38 \mathrm{sec}$. Moreover, when the size of graphs grows up to 4977 vertices (46 input schemas), the linear program found a solution in $533,95 \mathrm{sec} \rightsquigarrow$ $9,23 \mathrm{mn}$, which is a reasonable automatic resolution time with respect to time users need to do such a tedious task manually.

To conclude, our approach LP4HM is able to match N SOD graphs in an affordable time for an important size of structural graphs.

\section{CONCLUSiON}

In this paper, we presented an integer linear program, named LP4HM, performing holistic Statistical Open Data (SOD) graph matching. This program is part of our graphbased ETL approach proposed to warehouse SOD. LP4HM is and extension of the weighted graph matching problem with different constraints on graph structures and matching setup, especially 1:1 matching. We have performed a useroriented benchmark on LP4HM and some other approaches 
in the literature. The comparative experimentations show the effectiveness of LP4HM without threshold tuning and the LP4HM(relax) that is a relaxation of our model able to resolve complex matching cardinalities. Moreover, we have demonstrated the importance of globally optimal solutions compared to locally optimal solutions in a holistic case. LP4HM shows a reasonable resolution time for several input graphs.

For future work, we will extend our linear program with some constraints related to properties of graph labels. We aim to handle the specificities of ontologies in our model. Furthermore, some algorithms in the combinatorial optimisation field will be tested in order to make a comparative study on the performance of our model on very large problems.

\section{REFERENCES}

[1] A. Berro, I. Megdiche, and O. Teste, "A content-driven ETL processes for open data," in New Trends in Database and Information Systems II, Selected papers of the 18th East European Conference on Advances in Databases and Information Systems, ADBIS'14. Springer International Publishing, 2014, vol. 312, pp. 29-40.

[2] E. Rahm and P. A. Bernstein, "A survey of approaches to automatic schema matching," VLDB JOURNAL, vol. 10, 2001.

[3] R. Angles and C. Gutierrez, "Survey of graph database models," $A C M$ Comput. Surv., vol. 40, no. 1, p. 139, 2008.

[4] A. Berro, I. Megdiche, and O. Teste, "Graph-based etl processes for warehousing statistical open data," in ICEIS 2015, 2015, pp. 271-278.

[5] F. Ravat, O. Teste, R. Tournier, and G. Zurfluh, "Graphical querying of multidimensional databases," in ADBIS 2007, 2007, pp. 298-313.

[6] E. Malinowski and E. Zimányi, "Hierarchies in a multidimensional model: From conceptual modeling to logical representation," Data Knowl. Eng., vol. 59, no. 2, pp. 348-377, 2006.

[7] E. Rahm, "Towards large-scale schema and ontology matching," in Schema Matching and Mapping, ser. Data-Centric Systems and Applications, Z. Bellahsene, A. Bonifati, and E. Rahm, Eds. Springer Berlin Heidelberg, 2011, pp. 3-27.

[8] P. Shvaiko and J. Euzenat, "A survey of schema-based matching approaches," in Journal on Data Semantics IV. Springer Berlin Heidelberg, 2005, pp. 146-171.

[9] B. He and K. C. Chang, "Automatic complex schema matching across web query interfaces: A correlation mining approach," ACM Trans. Database Syst., vol. 31, no. 1, pp. 346-395, 2006.

[10] W. Su, J. Wang, and F. H. Lochovsky, "Holistic schema matching for web query interfaces," in Advances in Database Technology EDBT 2006, 10th International Conference on Extending Database Technology, Munich, Germany, March 26-31, 2006, Proceedings, 2006, pp. $77-94$.

[11] K. Saleem, Z. Bellahsene, and E. Hunt, "Performance oriented schema matching." in DEXA, vol. 4653, 2007, pp. 844-853.

[12] A. Benharkat, R. Rifaieh, S. Sellami, M. Boukhebouze, and Y. Amghar, "PLASMA: A platform for schema matching and management," IBIS, vol. 5, pp. 9-20, 2007.

[13] A. Termier, M.-C. Rousset, and M. Sebag, "Dryade: a new approach for discovering closed frequent trees in heterogeneous tree databases," in Data Mining, 2004. ICDM '04. Fourth IEEE International Conference on, Nov 2004, pp. 543-546.

[14] U. Chukmol, R. Rifaieh, and N. Benharkat, "Exsmal: Edi/xml semiautomatic schema matching algorithm," in E-Commerce Technology, 2005. CEC 2005. Seventh IEEE International Conference on, July 2005, pp. $422-425$.

[15] D. Aumueller, H.-H. Do, S. Massmann, and E. Rahm, "Schema and ontology matching with coma++," in SIGMOD '05, 2005, pp. 906-908.

[16] S. Melnik, H. Garcia-Molina, and E. Rahm, "Similarity flooding: A versatile graph matching algorithm and its application to schema matching," in Proceedings of the 18th International Conference on Data Engineering, ser. ICDE '02. IEEE Computer Society, 2002, pp. 117-.
[17] F. Duchateau, Z. Bellahsene, and M. Roche, "Bmatch: a semantically context-based tool enhanced by an indexing structure to accelerate schema matching," in 23èmes Journées Bases de Données Avancées, BDA 2007, Marseille, 23-26 Octobre 2007, Actes (Informal Proceedings), 2007.

[18] J. Huber, T. Sztyler, J. Nner, and C. Meilicke, "Codi: Combinatorial optimization for data integration: results for oaei 2011." in $O M$, ser. CEUR Workshop Proceedings, vol. 814. CEUR-WS.org, 2011.

[19] J. Euzenat and P. Valtchev, "Similarity-based ontology alignment in owl-lite," in Proc. 16th european conference on artificial intelligence (ECAI), Valencia (ES). IOS press, 2004, pp. 333-337.

[20] J. Euzenat and P. Shvaiko, Ontology matching, 2nd ed. Heidelberg (DE): Springer-Verlag, 2013.

[21] M. Niepert, C. Meilicke, and H. Stuckenschmidt, "A ProbabilisticLogical Framework for Ontology Matching," in Proceedings of the 24th AAAI Conference on Artificial Intelligence. AAAI Press, 2010, pp. 1413-1418.

[22] A. Schrijver, Combinatorial Optimization - Polyhedra and Efficiency. Springer, 2003.

[23] H. A. Almohamad and S. Duffuaa, "A linear programming approach for the weighted graph matching problem," IEEE Trans. Pattern Anal. Mach. Intell, pp. 522-525, 1993.

[24] J. Edmonds, "Maximum matching and a polyhedron with 0, 1-vertices," Journal of Research of the National Bureau of Standards B, vol. 69, pp. 125-130, 1965.

[25] Z. Wu and M. Palmer., "Verb semantics and lexical selection." in In 32nd. Annual Meeting of the Association for Computational Linguistics, 1994, pp. 133-138.

[26] D. Lin, "An information-theoretic definition of similarity" in In Proceedings of the 15th International Conference on Machine Learning. Morgan Kaufmann, 1998, pp. 296-304.

[27] F. Plastria, "Formulating logical implications in combinatorial optimisation," European Journal of Operational Research, vol. 140, no. 2, pp. $338-353,2002$.

[28] F. Duchateau and Z. Bellahsene, "Designing a benchmark for the assessment of schema matching tools," Open Journal of Databases $(O J D B)$, vol. 1, no. 1, pp. 3-25, 2014. 